\title{
Carotid Plaque, Carotid Intima-Media Thickness, and Coronary Calcification Equally Discriminate Prevalent Cardiovascular Disease in Kidney Disease
}

\author{
Gbemisola A. Adeseun $^{a}$ Dawei Xie $^{b}$ Xin Wang ${ }^{b}$ Marshall M. Joffe ${ }^{b}$ \\ Emile R. Mohler III ${ }^{c}$ Raymond R. Townsend ${ }^{d}$ Matthew Budoffe Sylvia E. Rosas ${ }^{d, f}$ \\ ${ }^{a}$ Renal Division, Department of Medicine, University of Southern California Keck School of Medicine, ${ }^{b}$ Department of \\ Biostatistics and Epidemiology, Center for Clinical Epidemiology and Biostatistics, University of Pennsylvania, ' ${ }^{\text {SSection }}$ \\ of Vascular Medicine, Cardiovascular Division, and ${ }^{\mathrm{d}}$ Renal, Electrolyte and Hypertension Division, Department \\ of Medicine, University of Pennsylvania School of Medicine, and e David Geffen School of Medicine, University of \\ California, Los Angeles, Calif., ${ }^{\text {} P h i l a d e l p h i a ~ V e t e r a n s ~ A d m i n i s t r a t i o n ~ M e d i c a l ~ C e n t e r, ~ P h i l a d e l p h i a, ~ P a ., ~ U S A ~}$
}

\section{Key Words}

Carotid intima-media thickness $\cdot$ Coronary artery

calcification $\cdot$ Kidney $\cdot$ Plaque

\begin{abstract}
Background: Despite the significant morbidity and mortality attributable to cardiovascular disease (CVD), risk stratification remains an important challenge in the chronic kidney disease (CKD) population. We examined the discriminative ability of noninvasive measures of atherosclerosis, including carotid intima-media thickness (cIMT), carotid plaque, coronary artery calcification (CAC) and ascending and descending thoracic aorta calcification (TCAC), and Framingham risk score (FRS) to predict self-reported prevalent CVD. Methods and Results: Participants were enrolled in the cIMT ancillary study of the Chronic Renal Insufficiency Cohort (CRIC) study and also had all of the above measures within an 18-month period. CVD was present in $21 \%$ of study participants. C-statistics were used to ascertain the discriminatory power of each measure of atherosclerosis. The study population $(n=$ 220 ) was $64 \%$ male; $51 \%$ black and $45 \%$ white. The proportion of individuals with estimated glomerular filtration rate
\end{abstract}

$\geq 60,45-59,30-44$, and $<30 \mathrm{ml} / \mathrm{min} / 1.73 \mathrm{~m}^{2}$ was $21,41,28$, and $11 \%$, respectively. In multivariable analyses adjusting for demographic factors, we failed to find a difference between CAC, carotid plaque, and CIMT as predictors of self-reported prevalent CVD (C-statistic 0.70, 95\% Cl: 0.62-0.78; C-statistic $0.68,95 \%$ Cl: $0.60-0.75$, and C-statistic $0.64, \mathrm{Cl}: 0.56-0.72$, respectively). CAC was statistically better than FRS. FRS was the weakest discriminator of self-reported prevalent CVD (C-statistic 0.58). Conclusions: There was a significant burden of atherosclerosis among individuals with CKD, ascertained by several different imaging modalities. We were unable to find a difference in the ability of CAC, carotid plaque, and CIMT to predict self-reported prevalent CVD.

Copyright $\odot 2012$ S. Karger AG, Basel

\section{Introduction}

Individuals with chronic kidney disease (CKD) are at extraordinarily high risk of adverse cardiovascular events [1]. The reasons for this excess cardiovascular risk are manifold and likely related to a CKD milieu that promotes vascular calcification and atherogenesis. Beyond

\section{KARGER}

Fax +4161306 1234 E-Mail karger@karger.ch www.karger.com (c) 2012 S. Karger AG, Basel

$0250-8095 / 12 / 0364-0342 \$ 38.00 / 0$

Accessible online at:

www.karger.com/ajn
Dr. Sylvia E. Rosas

Renal, Electrolyte and Hypertension Division

1st Floor Founders Building, 3400 Spruce Street

Philadelphia, PA 19104 (USA)

E-Mail sylvia.rosas@uphs.upenn.edu 
traditional cardiovascular risk factors, such as hypertension, diabetes, and dyslipidemia, CKD is characterized by an inflammatory state that has been proposed to be an independent risk factor for atherosclerosis [2]. CKD is also associated with hyperphosphatemia, increased oxidative stress, and a decrease in vascular calcification inhibitors, resulting in upregulation of pathways that favor vascular smooth muscle transformation into osteoblastlike cells, resulting in vascular calcification [3].

Vascular calcification is exceedingly common in CKD and can occur in the intima and/or media of blood vessels in multiple vascular beds [4]. Vascular calcification of the media, also common in diabetes, leads to increased arterial stiffness, increased pulse wave velocity, and left ventricular hypertrophy [5]. Alternatively, intimal vascular calcification occurs in both the CKD and non-CKD population and is directly related to atherosclerosis and ischemic cardiovascular disease (CVD) [6] .

Noninvasive methods such as carotid ultrasound and $\mathrm{CT}$ are used to quantify atherosclerosis and determine the presence of vascular calcification. Coronary artery calcification (CAC) correlates with obstructive coronary artery disease in both the general and CKD population $[7,8]$. CKD is a risk factor for the presence of CAC, as well as CAC progression $[9,10]$. While carotid intima-media thickness (cIMT) and CAC predict adverse cardiovascular events $[11,12]$, CAC is a more robust predictor of coronary events while cIMT may be a better predictor of stroke in the general population [13]. Less is known about the predictive utility of thoracic aorta calcification and carotid plaque, which are emerging markers of increased cardiovascular risk and morality [14].

To date, no published study has compared several different measures of cardiovascular risk stratification in a population with CKD. Therefore, we assessed the ability of CAC, cIMT, carotid plaque, and ascending and descending thoracic aorta calcification (TCAC) to discern prevalent CVD in CKD.

\section{Methods}

\section{Study Design}

This is a cross-sectional study of noninvasive measures of atherosclerosis as part of the cIMT ancillary study of the Chronic Renal Insufficiency Cohort (CRIC). The CRIC has been described in detail [15]. In brief, the CRIC is a multicenter prospective cohort study comprised of 3,939 patients with CKD, recruited from 7 centers in the United States. CRIC cIMT participants were recruited from 4 of 7 CRIC sites with expertise in cardiovascular imaging technology. Recruitment for the CRIC IMT ancillary started a year after recruitment for the main CRIC started, and participants had a carotid ultrasound performed at their convenience during any of their annual CRIC visits. CRIC participants are aged 21-74 years with an estimated glomerular filtration rate $20-70 \mathrm{ml} / \mathrm{min} / 1.73 \mathrm{~m}^{2}$ at the screening visit as calculated by the abbreviated Modification of Diet in Renal Disease (MDRD) equation. Detailed medical histories and anthropometric and blood pressure measurements were obtained. Participants also underwent an assessment of subclinical CVD. Individuals with a history of coronary artery bypass grafting were excluded from CT study after 2005. Two hundred and twenty participants who underwent both cIMT and CT within an 18 -month time frame were included in this report. The mean time between studies was 252 days with a median of 293 days. Physiological markers such as creatinine and proteinuria were measured at each CRIC annual visit, and the results from the visit nearest to the carotid ultrasound visit were used for this analysis. Written informed consent was obtained from all study participants. The CRIC and the CRIC IMT ancillary studies were approved by the institutional review boards from each institution.

Prevalent CVD was defined as a self-reported history of myocardial infarction or revascularization, peripheral artery disease, and/or stroke. Participants were also asked to report hospitalizations during subsequent visits. Participants also had the opportunity to update their cardiovascular history during subsequent study visits, and the most up-to-date information regarding CVD status was used in analyses. There was no independent adjudication of the medical history obtained from participants. The Framingham risk score (FRS) was based on the risk prediction model detailed by D'Agostino et al. [16]. The calculated 10-year FRS was then categorized $(<10,10$ to $<20$, and $\geq 20 \%)$.

\section{Carotid Ultrasound}

To ascertain cIMT and presence of carotid plaque, participants underwent carotid ultrasound. Carotid ultrasonographic images were obtained using a standardized linear array $7.5-\mathrm{mHz}$ probe in the ECG-gated B mode according to standardized protocols. Interpretation of the carotid artery images was performed using an FDA-approved software package (Medical Imaging Applications, Iowa City, Iowa, USA). Using the carotid bulb as an anatomic reference, measurements were taken of the far wall of the common carotid artery as this location provides the most consistent readings, with the carotid bulb serving as an anatomic reference. A carotid plaque was defined as a focal region with CIMT $>1.5 \mathrm{~mm}$ that protrudes into the lumen and that is distinct from the adjacent boundary [17].

\section{Computed Tomography}

Participants underwent two electron-beam CTs (EBCT) or multidetector CT examination for CAC and TCAC quantification as previously described. EBCTs were performed on a C-150 Imatron scanner (GE, San Francisco, Calif., USA) according to established protocol. CAC was performed in a random sample who were eligible for the procedure. Approximately a third of participants had CT. CAC, ascending TCAC, and descending TCAC were scored separately using the Agatston method. EBCTs were scored by a single reader for consistency. The EBCT score that was closest in proximity to the carotid ultrasound examination was used for analyses. 
Table 1. Association of the different subclinical cardiovascular measures with CAC scores in participants without self-reported CVD

\begin{tabular}{|c|c|c|c|c|}
\hline \multirow[t]{2}{*}{ Variable } & \multicolumn{4}{|l|}{ CAC } \\
\hline & $0(n=63)$ & 0 to $<100(\mathrm{n}=57)$ & 100 to $<400(\mathrm{n}=41)$ & $\geq 400(n=59)$ \\
\hline \multicolumn{5}{|l|}{ Carotid plaque } \\
\hline Mean $\pm S D$ & $0.6 \pm 1.2$ & $0.7 \pm 1.1$ & $1.3 \pm 1.4$ & $2.3 \pm 1.8$ \\
\hline Median (P25-P75) & $0.0(0.0-1.0)$ & $0.0(0.0-1.0)$ & $1.0(0.0-2.0)$ & $2.0(0.0-4.0)$ \\
\hline \multicolumn{5}{|l|}{ cIMT } \\
\hline Mean \pm SD & $0.7 \pm 0.1$ & $0.8 \pm 0.2$ & $0.8 \pm 0.2$ & $0.8 \pm 0.2$ \\
\hline Median (P25-P75) & $0.7(0.6-0.8)$ & $0.8(0.7-0.9)$ & $0.8(0.6-0.9)$ & $0.8(0.7-1.0)$ \\
\hline \multicolumn{5}{|l|}{ Ascending TCAC } \\
\hline Mean \pm SD & $1.7 \pm 12.3$ & $46.4 \pm 263.9$ & $15.8 \pm 59.0$ & $26.4 \pm 96.8$ \\
\hline Median (P25-P75) & $0.0(0.0-0.0)$ & $0.0(0.0-0.0)$ & $0.0(0.0-0.0)$ & $0.0(0.0-0.0)$ \\
\hline \multicolumn{5}{|l|}{ Descending TCAC } \\
\hline Mean \pm SD & $60.2 \pm 246.7$ & $89.8 \pm 246.8$ & $382.0 \pm 578.1$ & $831.5 \pm 2,106.6$ \\
\hline Median (P25-P75) & $0.0(0.0-0.0)$ & $0.0(0.0-53.3)$ & $80.3(0.0-757.4)$ & $75.6(0.0-678.3)$ \\
\hline \multicolumn{5}{|c|}{ 10-year Framingham risk } \\
\hline Mean $\pm \mathrm{SD}$ & $12.4 \pm 8.6$ & $19.2 \pm 9.5$ & $21.4 \pm 9.0$ & $23.2 \pm 7.5$ \\
\hline Median (P25-P75) & $11.2(4.7-18.4)$ & $18.5(11.2-30.0)$ & $24.8(13.7-30.0)$ & $25.3(18.4-30.0)$ \\
\hline
\end{tabular}

\section{Statistical Analysis}

Descriptive statistics were performed using means with standard deviation and medians with interquartile ranges for continuous variables and proportions for categorical variables.

The distribution of cIMT, plaque, CAC, ascending TCAC, and descending TCAC were not normally distributed and thus were analyzed as categorical variables. A substantial portion of individuals had no ascending or descending TCAC, so these variables were categorized thus: 0,1 to $<$ median, and $\geq$ median. CAC was categorized as follows: 0,1 to $<100,100$ to $<400, \geq 400$ Agatston units.

To assess the degree of variance in the prevalence of CVD that can be explained by the presence of traditional atherosclerotic risk factors in the study population and in the subgroups defined above, the discriminative power of the models was assessed using the C-statistics and their 95\% CI obtained using bootstrapping techniques. A multivariate logistic regression model including demographic variables (age, gender, and race) to obtain an adjusted C-statistic was performed. The statistical software package SAS version 9.2 was used for all analyses (SAS Institute Inc., Cary, N.C., USA).

\section{Results}

Demographic and comorbidity data are presented in online supplementary table 1 (for all online suppl. material, see www.karger.com/doi/10.1159/000342794). The average age was 61.6 years, with a predominance of men (64\%). Approximately half of the study population was black. Although the self-reported prevalence of CVD was $20 \%$, risk factors for CVD were common with hyperten- sion and diabetes affecting 90 and $55 \%$ of the population, respectively. The metabolic syndrome was present in 55\% of participants, a marker of increased CVD risk. Unadjusted analyses revealed a statistically significant association between estimated glomerular filtration rate with CAC and cIMT (online suppl. table 2).

We found that several subclinical measures had increased values as the CAC scores increased in participants without self-reported CVD (table 1). This was more evident with carotid plaque and descending TCAC.

In unadjusted analyses, the ability of CAC, carotid plaque, and cIMT to discriminate prevalent CVD were comparable with C-statistics of $0.67,0.64$, and 0.61 , respectively $(\mathrm{p}>0.05)$. Ascending and descending TCACs with C-statistics of 0.54 and 0.57 , respectively. The FRS has been validated in the general population but had marginal discriminatory value in this setting, with a C-statistic of only 0.56 (table 2).

After adjusting for age, gender, and race in multivariable logistic regression analyses, the discriminatory power of each measure of CVD risk improved. CAC appeared to be the best predictor of self-reported prevalent CVD followed by carotid plaque, with C-statistics of the adjusted models 0.70 and 0.68 , respectively. The C-statistics for ascending and descending TCAC also improved modestly. FRS was the least discriminatory (C-statistic 0.58). Differences between CAC, carotid plaque, and cIMT were not statistically significant. 
Table 2. Association between measures of atherosclerosis and prevalent CVD

\begin{tabular}{lllll}
\hline Measurement & $\begin{array}{l}\text { Unadjusted } \\
\text { C-statistic }\end{array}$ & CI & $\begin{array}{l}\text { Adjusted } \\
\text { C-statistic }\end{array}$ & CI \\
\hline CAC & 0.67 & $0.59-0.76$ & 0.70 & $0.62-0.78$ \\
Carotid plaque & 0.64 & $0.56-0.73$ & 0.68 & $0.60-0.75$ \\
cIMT & 0.61 & $0.53-0.69$ & 0.63 & $0.55-0.71$ \\
Ascending TCAC & 0.54 & $0.49-0.59$ & 0.64 & $0.56-0.71$ \\
Descending TCAC & 0.57 & $0.49-0.66$ & 0.61 & $0.52-0.69$ \\
10-year Framingham risk & 0.56 & $0.47-0.66$ & 0.58 & $0.47-0.68$ \\
\hline
\end{tabular}

${ }^{1}$ Adjusted for age, gender, and race.

\section{Discussion}

This study evaluates different measures of cardiovascular risk stratification including CAC, ascending and descending TCAC, cIMT, carotid plaque, and the FRS in a diverse cohort of individuals with CKD. Using these different imaging modalities, we identified a high burden of atherosclerosis among individuals with CKD. Of the noninvasive direct measures of atherosclerosis, we were unable to detect any statistical difference between CAC and carotid plaque or CIMT as predictors of self-reported prevalent CVD.

Noninvasive measures of atherosclerosis are increasingly used in the CKD population for cardiovascular risk stratification [18]. It is postulated that both traditional and nontraditional risk factors contribute to the early and extensive $\mathrm{CAC}$ that has been observed among individuals with CKD [10]. CAC has several advantages which include an established algorithm, wide availability, and lack of dependence on the operator. Measurement of cIMT, on the other hand, is operator dependent and requires specialized equipment and training. In the Multiethnic Study of Atherosclerosis in individuals without self-reported CVD, CAC was superior to CIMT for prediction of any incident CVD or any coronary event [13]. However in the same study, cIMT was a better predictor of stroke. Prospective data regarding the role of noninvasive measures of atherosclerosis and cardiovascular risk stratification are limited in the nondialysis-dependent CKD population. There is evidence that there is a direct correlation between CAC score and obstructive luminal lesions within coronary arteries in patients with CKD [8]. CAC is superior to IMT in predicting self-reported prevalent CVD identified by coronary angiography [19]. Further, CAC is associated with increased cardiovascular events and hos- pitalizations [18]. Although we found that CAC is associated with the highest index of discrimination for self-reported prevalent CVD which improved with the inclusion of demographic variables, we were unable to find a statistical difference with the other subclinical measures to predict self-reported prevalent CVD in our population. This improvement after adjustment reflects confounding by age, gender, and race, variables that have been associated with CAC in other studies [9].

We did not find a statistically significant difference in the discriminative power of CAC, carotid plaque, and cIMT. Prior research has shown that carotid plaque and cIMT are highly correlated, and each independently predicts cardiovascular events in non-CKD cohorts [20]. To date, there are no studies in the CKD population that compare CIMT and plaque prediction of CVD, although the CRIC study is poised to address this question in the future. A prospective study of individuals with ESRD found that carotid plaque progression, and not IMT, was an important predictor of cardiovascular events suggesting that plaque is a more sensitive measure of atherosclerosis than cIMT [21]. In our study, we found that the Cstatistic for carotid plaque was higher than that of cIMT in both univariable and multivariable analyses; however, this difference was not statistically significant. We may not have been sufficiently powered to detect a difference between carotid plaque and IMT. Nonetheless, our results indicate that the use of carotid plaque and cIMT would be reasonable alternatives to CAC. There is increasing concern about the use of radiation with CT. Therefore, our results demonstrate that carotid ultrasound may be a reasonable alternative. In addition, particularly in younger individuals (20-30 years) who typically do not have calcification, carotid ultrasound is likely the more appropriate subclinical cardiovascular tool. 
Aortic arch calcification is prevalent in CKD [18] and presence of aortic arch calcification is associated with other markers of increased cardiovascular risk [22]. We found that neither ascending nor descending aortic calcification was a strong predictor of self-reported prevalent CVD in unadjusted analyses with only mild improvement in predictive power with multivariable analyses.

In addition to noninvasive measures of atherosclerosis, clinical cardiovascular risk prediction tools such as the FRS can be an effective means of cardiovascular risk stratification in the general population. In our study population, the FRS was marginally associated with self-reported prevalent CVD. Weiner et al. [23] previously described a similar C-statistic for incident cardiovascular events predicted by the FRS among men in a CKD cohort (10-year C-statistics: 0.60 and 0.73 for men and women, respectively). In comparison, our study population was comprised of significantly more diabetic participants and a higher proportion of blacks, which may account for the difference. Adjusting for demographic variables did not have a significant impact on the $\mathrm{C}$-statistic, likely because both age and gender are included in the Framingham risk calculation.

There are important limitations of this study. First, we relied on participant self-report of CVD, which may have introduced misclassification bias. It is well known that several factors impact patient knowledge of CVD. Patients may underestimate or overestimate their degree of cardiovascular risk [24]. Lack of knowledge about comorbidities has been associated with increased mortality in a dialysis cohort [25]. Misclassification bias may also be present as a result of the possible lag between determination of prevalent CVD and subsequent cardiovascular imaging. Second, our study was performed in a subset of CRIC clinical sites and therefore the sample size is limited. The results of this study may not be generalizable to other populations. Participants in the CRIC study are not representative of the general CKD population. Study participants were predominantly male with predominantly stage $3 \mathrm{CKD}$, a relative low level of proteinuria, and good blood pressure control. Thus, selection bias may have resulted in enrollment of a comparatively healthier subset of individuals with CKD, limiting the generalizability of our findings. Finally, we are not able to evaluate the predictive utility of each of these tests in measuring incident cardiovascular events due to limited follow-up.

In conclusion, subclinical cardiovascular measures identify a significant burden of disease in CKD. CAC appears to be the best predictor of self-reported prevalent
CVD. However, no statistical difference was found with cIMT or carotid plaque. Carotid plaque and cIMT are appealing in that radiation is not required to obtain these measures. Given the technical expertise required to obtain IMT, the presence of carotid plaque is emerging as an important tool for cardiovascular risk stratification in CKD. Prospective studies such as CRIC will allow us to determine the predictive value of these risk assessment tools for incident cardiovascular events and mortality.

\section{Acknowledgments}

We thank all participants and investigators of the CRIC Study for their contributions. G.A.A. was supported by a research supplement to S.E.R.'s National Institutes of Health (NIH) grant R01 DK080033. S.E.R. was supported by NIH grant DK-064343 and R01 DK080033 from the National Institute of Diabetes and Digestive and Kidney Diseases and by the Department of Veterans Affairs (HSR \& D IIR 05-247).

Funding for the CRIC Study was obtained under a cooperative agreement from the National Institute of Diabetes and Digestive and Kidney Diseases (U01DK060990, U01DK060984, U01DK061022, U01DK061021, U01DK061028, U01DK060980, U01DK060963, and U01DK060902). In addition, this work was supported in part by the University of Pennsylvania CTRC CTSA UL1 RR-024134, Johns Hopkins University UL1 RR-025005, Michigan Institute for Clinical and Health Research (MICHR) UL1RR024986, and the Clinical and Translational Research, Education, and Commercialization Project (CTRECP).

\section{Disclosure Statement}

None of the authors have any conflicts of interest to disclose.

References

$$
\begin{aligned}
& \text { Foley RN, Parfrey PS, Sarnak MJ: Clinical } \\
& \text { epidemiology of cardiovascular disease in } \\
& \text { chronic renal disease. Am J Kidney Dis 1998; } \\
& \text { 32:S112-S119. } \\
& \text { Lemos MM, Jancikic AD, Sanches FM, et al: } \\
& \text { Intima-media thickness is associated with } \\
& \text { inflammation and traditional cardiovascu- } \\
& \text { lar risk factors in non-dialysis-dependent } \\
& \text { patients with chronic kidney disease. Neph- } \\
& \text { ron Clin Pract 2010;115:c189-c194. } \\
& \text { Giachelli CM: The emerging role of phos- } \\
& \text { phate in vascular calcification. Kidney Int } \\
& \text { 2009;75:890-897. } \\
& 4 \text { London GM, Guerin AP, Marchais SJ, Me- } \\
& \text { tivier F, Pannier B, Adda H: Arterial media } \\
& \text { calcification in end-stage renal disease: im- } \\
& \text { pact on all-cause and cardiovascular mortal- } \\
& \text { ity. Nephrol Dial Transplant 2003;18:1731- } \\
& \text { 1740. } \\
& \text { Chen NX, Moe SM: Arterial calcification in } \\
& \text { diabetes. Curr Diab Rep 2003;3:28-32. }
\end{aligned}
$$

Adeseun/Xie/Wang/Joffe/Mohler III/ Townsend/Budoff/Rosas 
6 Nakamura S, Ishibashi-Ueda H, Niizuma S, Yoshihara F, Horio T, Kawano Y: Coronary calcification in patients with chronic kidney disease and coronary artery disease. Clin J Am Soc Nephrol 2009;4:1892-1900.

7 Greenland P, Bonow RO, Brundage BH, et al: ACCF/AHA 2007 clinical expert consensus document on coronary artery calcium scoring by computed tomography in global cardiovascular risk assessment and in evaluation of patients with chest pain: a report of the American College of Cardiology Foundation Clinical Expert Consensus Task Force (ACCF/AHA Writing Committee to Update the 2000 Expert Consensus Document on Electron Beam Computed Tomography). Circulation 2007;115:402-426.

$\checkmark 8$ Robinson J, Tan AU, Wilensky RL, Matthai W, Munoz M, Rosas SE: Electron-beam computerized tomography correlates with coronary angiogram in chronic kidney disease patients. Am J Nephrol 2007;27:247-252.

$\checkmark 9$ Kestenbaum BR, Adeney KL, de Boer IH, Ix JH, Shlipak MG, Siscovick DS: Incidence and progression of coronary calcification in chronic kidney disease: the Multi-Ethnic Study of Atherosclerosis. Kidney Int 2009; 76:991-998

$\checkmark 10$ Kramer H, Toto R, Peshock R, Cooper R, Victor R: Association between chronic kidney disease and coronary artery calcification: the Dallas Heart Study. J Am Soc Nephrol 2005;16:507-513.

-11 Hodis HN, Mack WJ, LaBree L, et al: The role of carotid arterial intima-media thickness in predicting clinical coronary events. Ann Intern Med 1998;128:262-269.
12 Chambless LE, Heiss G, Folsom AR, et al: Association of coronary heart disease incidence with carotid arterial wall thickness and major risk factors: the Atherosclerosis Risk in Communities (ARIC) Study, 19871993. Am J Epidemiol 1997;146:483-494.

13 Folsom AR, Kronmal RA, Detrano RC, et al: Coronary artery calcification compared with carotid intima-media thickness in the prediction of cardiovascular disease incidence: the Multi-Ethnic Study of Atherosclerosis (MESA). Arch Intern Med 2008;168. 1333-1339.

14 Rundek T, Arif H, Boden-Albala B, Elkind MS, Paik MC, Sacco RL: Carotid plaque, a subclinical precursor of vascular events: the Northern Manhattan Study. Neurology 2008;70:1200-1207.

15 Feldman HI, Appel LJ, Chertow GM, et al: The Chronic Renal Insufficiency Cohort (CRIC) Study: design and methods. J Am Soc Nephrol 2003;14:S148-S153.

16 D'Agostino RB Sr, Vasan RS, Pencina MJ, et al: General cardiovascular risk profile for use in primary care: the Framingham Heart Study. Circulation 2008;117:743-753.

-17 Touboul PJ, Hennerici MG, Meairs S, et al: Mannheim intima-media thickness consensus. Cerebrovasc Dis 2004;18:346-349.

18 Watanabe R, Lemos MM, Manfredi SR, Draibe SA, Canziani ME: Impact of cardiovascular calcification in nondialyzed patients after 24 months of follow-up. Clin J Am Soc Nephrol 2010;5:189-194.
19 Terry JG, Carr JJ, Tang R, et al: Coronary artery calcium outperforms carotid artery intima-media thickness as a noninvasive index of prevalent coronary artery stenosis. Arterioscler Thromb Vasc Biol 2005;25:17231728

20 Bots ML, Hoes AW, Koudstaal PJ, Hofman A, Grobbee DE: Common carotid intimamedia thickness and risk of stroke and myocardial infarction: the Rotterdam Study. Circulation 1997;96:1432-1437.

$>21$ Benedetto FA, Tripepi G, Mallamaci F, Zoccali C: Rate of atherosclerotic plaque formation predicts cardiovascular events in ESRD. J Am Soc Nephrol 2008;19:757-763.

22 Iijima K, Hashimoto H, Hashimoto M, et al: Aortic arch calcification detectable on chest $\mathrm{X}$-ray is a strong independent predictor of cardiovascular events beyond traditional risk factors. Atherosclerosis 2010;210:137144.

23 Weiner DE, Tighiouart H, Elsayed EF, et al: The Framingham predictive instrument in chronic kidney disease. J Am Coll Cardiol 2007;50:217-224.

-24 Englert H, Müller-Nordhorn J, Seewald S, et al: Is patient self-report an adequate tool for monitoring cardiovascular conditions in patients with hypercholesterolemia? J Public Health (Oxf) 2010;32:387-394.

25 Cavanaugh KL, Merkin SS, Plantinga LC, Fink NE, Sadler JH, Powe NR: Accuracy of patients' reports of comorbid disease and their association with mortality in ESRD. Am J Kidney Dis 2008;52:118-127. 